\title{
A SARS-CoV-2 surrogate virus neutralization test based on antibody-mediated blockage of ACE2-spike protein-protein interaction
}

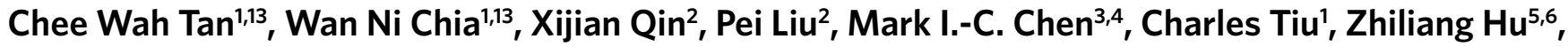 \\ Vivian Chih-Wei Chen ${ }^{1}{ }^{1}$, Barnaby E. Young ${ }^{10,7,8}$, Wan Rong Sia ${ }^{1}{ }^{1}$, Yee-Joo Tan ${ }^{9,10}$, Randy Foo', \\ Yongxiang Yi ${ }^{5}$, David C. Lye $e^{3,7,8,11}$, Danielle E. Anderson ${ }^{1,12 \bowtie}$ and Lin-Fa Wang ${ }^{1,12} \bowtie$
}

\begin{abstract}
A robust serological test to detect neutralizing antibodies to SARS-CoV-2 is urgently needed to determine not only the infection rate, herd immunity and predicted humoral protection, but also vaccine efficacy during clinical trials and after large-scale vaccination. The current gold standard is the conventional virus neutralization test requiring live pathogen and a biosafety level 3 laboratory. Here, we report a SARS-CoV-2 surrogate virus neutralization test that detects total immunodominant neutralizing antibodies targeting the viral spike (S) protein receptor-binding domain in an isotype- and species-independent manner. Our simple and rapid test is based on antibody-mediated blockage of the interaction between the angiotensin-converting enzyme 2 (ACE2) receptor protein and the receptor-binding domain. The test, which has been validated with two cohorts of patients with COVID-19 in two different countries, achieves $99.93 \%$ specificity and $95-100 \%$ sensitivity, and differentiates antibody responses to several human coronaviruses. The surrogate virus neutralization test does not require biosafety level 3 containment, making it broadly accessible to the wider community for both research and clinical applications.
\end{abstract}

T he COVID-19 outbreak was first recognized in December 2019 in Wuhan, China ${ }^{1}$ and has since spread to all parts of the world, resulting in a total of 10,357,662 confirmed infections with 508,055 deaths as of 1 July $2020^{2}$. The causative agent was identified as 2019-nCoV, subsequently designated SARS-CoV-2 ${ }^{3,4}$, which belongs to the species SARS-related coronavirus (SARSr-CoV), the same as for SARS-CoV, the causative agent of the SARS outbreak 17 years ago ${ }^{5}$.

Although molecular detection techniques, such as the polymerase chain reaction (PCR) and next-generation sequencing, have played an important role in acute diagnosis and monitoring of genetic changes of the virus, an urgent need exists for a reliable and versatile serological or antibody test. Such a test is needed for retrospective contact tracing, investigation of the asymptomatic infection rate, accurate determination of the case fatality rate, and assessment of herd immunity and humoral protective immunity in recovered patients and recipients of vaccine candidates, and in the search for the natural reservoir and intermediate host $(\mathrm{s})^{6}$. Research laboratories and pharmaceutical companies are racing to produce antibody tests that can detect COVID-19 infection with sufficient specificity and sensitivity ${ }^{6}$. There are two types of antibody test one can aim for. The first type is the conventional virus neutralization test (cVNT), which detects neutralizing antibodies (NAbs) in a patient's blood. The cVNT requires handling live SARS-CoV-2 in a specialized biosafety level 3 (BSL3) containment facility and is tedious and time-consuming, taking $2-4$ days to complete. The pseudovirus-based VNT (pVNT), on the other hand, can be performed in a BSL2 laboratory, but still requires the use of live viruses and cells ${ }^{7,8}$. All other assays, such as enzyme-linked immunosorbent assay (ELISA) and lateral flow assay (LFA) rapid tests, represent the second assay type, which detects total binding antibodies (BAbs) and is unable to differentiate between BAbs and NAbs ${ }^{6,9,10}$.

In this study, we established a surrogate VNT (sVNT) that detects NAbs, without the need for any live virus or cells, that can be completed in 1-2 $\mathrm{h}$ in a BSL2 laboratory. Using purified receptor-binding domain (RBD) from the $\mathrm{S}$ protein and the host cell receptor ACE2, our test is designed to mimic the virus-host interaction in an ELISA plate well. This RBD-ACE2 interaction can be neutralized (that is, blocked) by specific NAbs in patient or animal sera, in the same manner as in cVNT or pVNT.

\section{Results}

Biochemical simulation of virus-receptor interaction and antibody-mediated neutralization. Immediately after SARS-CoV-2 was identified as the causative agent of the COVID-19 outbreak, it was shown that human ACE2 (hACE2) is the main functional receptor for viral entry ${ }^{3}$. We hypothesized that the virus-receptor binding can be mimicked in vitro via a protein-protein interaction using purified recombinant hACE2 and the RBD of the SARS-CoV-2 S protein. This interaction can be blocked by virus NAbs present in

\footnotetext{
'Programme in Emerging Infectious Diseases, Duke-NUS Medical School, Singapore, Singapore. ${ }^{2}$ GenScript Biotech, Nanjing, China. ${ }^{3}$ National Centre for Infectious Diseases, Singapore, Singapore. ${ }^{4}$ Saw Swee Hock School of Public Health, National University of Singapore, Singapore, Singapore. ${ }^{5}$ Nanjing Infectious Disease Center, The Second Hospital of Nanjing, Nanjing University of Chinese Medicine, Nanjing, China. ${ }^{6}$ Center for Global Health, School of Public Health, Nanjing Medical University, Nanjing, China. ${ }^{7}$ Duke-NUS Medical School, Tan Tock Seng Hospital, Singapore, Singapore. ${ }^{8}$ Lee Kong Chian School of Medicine, Nanyang Technological University, Singapore, Singapore. 'Department of Microbiology and Immunology, Yong Loo Lin School of Medicine, National University Health System, National University of Singapore, Singapore, Singapore. ${ }^{10}$ Institute of Molecular and Cell Biology, Agency for Science, Technology and Research, Singapore, Singapore. "Yong Loo Lin School of Medicine, National University of Singapore, Singapore, Singapore. ${ }^{12}$ SingHealth Duke-NUS Global Health Institute, Singapore, Singapore. ${ }^{13}$ These authors contributed equally: Chee Wah Tan, Wan Ni Chia.

凶e-mail: Danielle.anderson@duke-nus.edu.sg; linfa.wang@duke-nus.edu.sg
} 
a

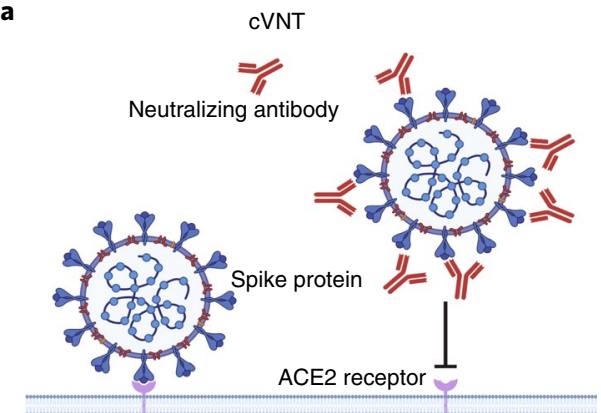

RBD of the spike protein binds to ACE2 receptor
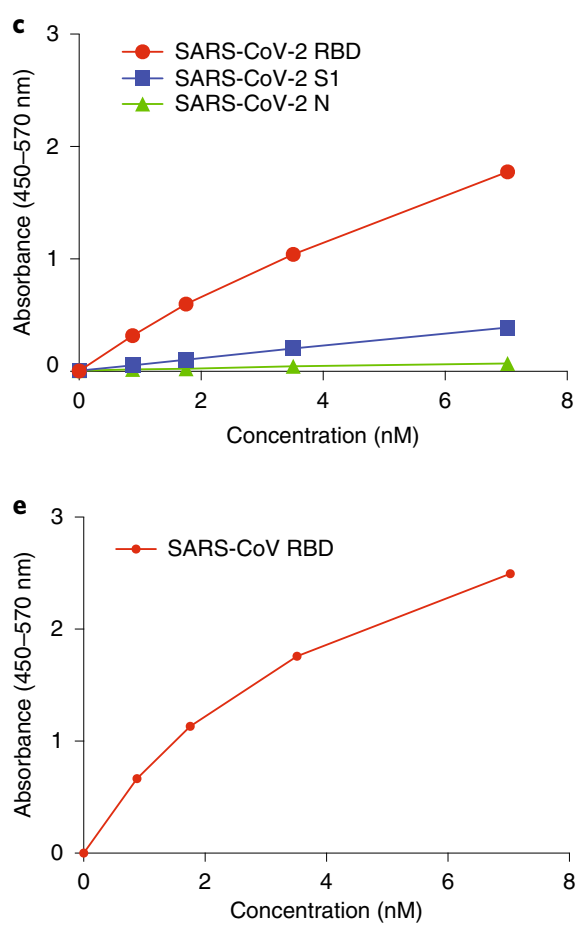

b

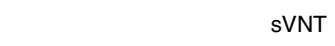

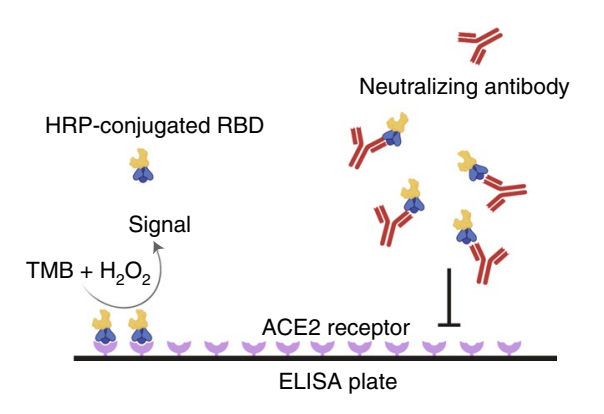

d

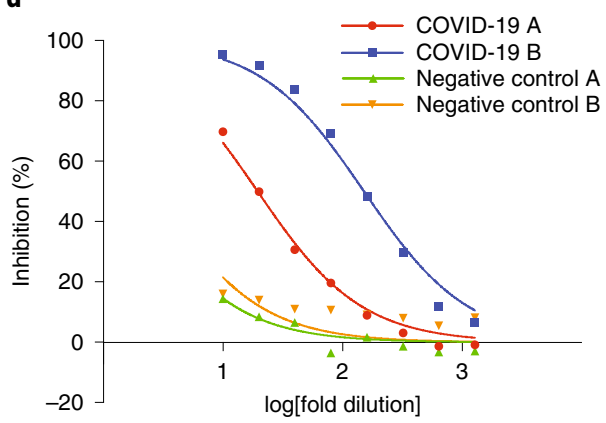

f

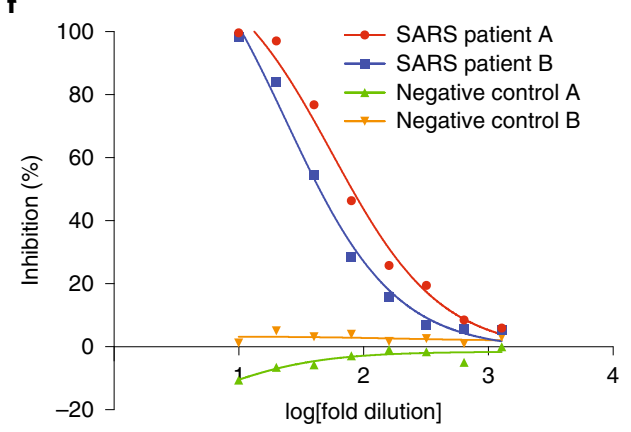

Fig. 1 | Principle and initial validation of the SARS-CoV-2 sVNT. a, The mechanism of cVNT. Anti-SARS-CoV-2 neutralizing antibodies block the SARS-CoV-2 spike protein from binding to hACE2 receptor proteins on the host cell surface. $\mathbf{b}$, In the sVNT assay, anti-SARS-CoV-2 neutralizing antibodies block HRP-conjugated RBD protein from binding to the hACE2 protein pre-coated on an ELISA plate. The illustrations were created using BioRender. c, Binding of HRP-conjugated SARS-CoV-2 N, S1 and RBD proteins to hACE2. d, Inhibition of SARS-CoV-2 RBD-hACE2 interaction by sera from patients with COVID-19. e, Binding of HRP-conjugated SARS-CoV RBD to hACE2. f, Inhibition of SARS-CoV RBD-hACE2 interaction by sera from patients with SARS. The data presented are the mean of two independent experiments.

the test serum, using the same principle as cVNT conducted using live virus inside a BSL3 facility (Fig. 1a,b).

In our study, direct binding was demonstrated using different SARS-CoV-2 proteins conjugated with horseradish peroxidase (HRP). There is a dose-dependent specific binding between hACE2 and the RBD or S1, but not the nucleocapsid $(\mathrm{N})$ protein, with the RBD producing the best binding characteristics (Fig. 1c). The HRP-RBD protein was chosen for subsequent studies. We then demonstrated that the specific RBD-hACE2 binding can be blocked or neutralized by COVID-19 sera in a dose-dependent manner, but not by sera from healthy controls (Fig. 1d). Using a panel of 20 convalescent sera, we demonstrated that HRP-RBD performed better in detecting NAbs than HRP-S1 (Extended Data Fig. 1). To prove that the same principle works with the closely related SARS-CoV, which also uses hACE2 as the entry receptor ${ }^{11}$, we repeated similar experiments and proved that the SARS-CoV RBD performed in an almost identical manner in the sVNT format (Fig. 1e,f). To further confirm that the sVNT is measuring true NAbs and can differentiate from BAbs detected by ELISA using the same RBD antigen, we compared the performance of 15 monoclonal antibodies (mAbs) from 4 different species ( 4 from mice, 4 from rabbits, 3 from llamas and 4 from humans) in RBD ELISA and RBD sVNT (Extended Data Fig. 2 and Supplementary Table 1). From each species, we have found $\mathrm{mAb}(\mathrm{s})$ with strong binding in ELISA, but weak or no neutralizing activity in sVNT.

Isotype- and species-independent neutralization. One of the advantages of sVNT is its ability to detect total RBD-targeting antibodies in patient sera, in contrast to most SARS-CoV-2 antibody tests published or marketed, most of which are isotype-specific, mainly for $\operatorname{IgM}$ or $\operatorname{IgG}$, with some for $\operatorname{Ig} \mathrm{A}^{9,10,12}$. From sera from convalescent patients with COVID-19 in Singapore, we designated four groups based on IgM or IgG ELISA levels, determined by our in-house capture ELISA assays (see Methods): high IgM/low IgG; low IgM/low IgG; low IgM/high IgG; and high IgM/high IgG. All groups showed strong neutralization activity in the sVNT (Fig. 2), 

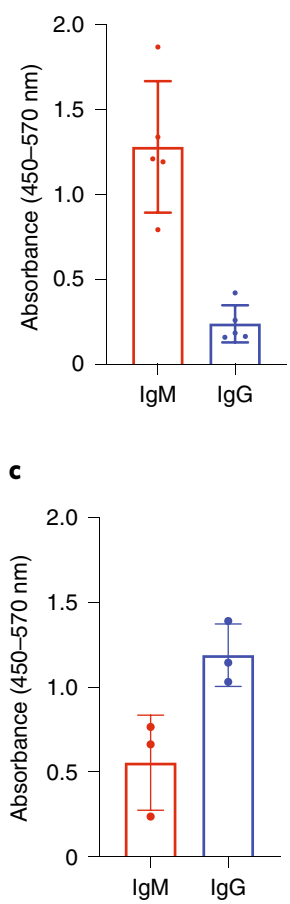

High IgM/low IgG

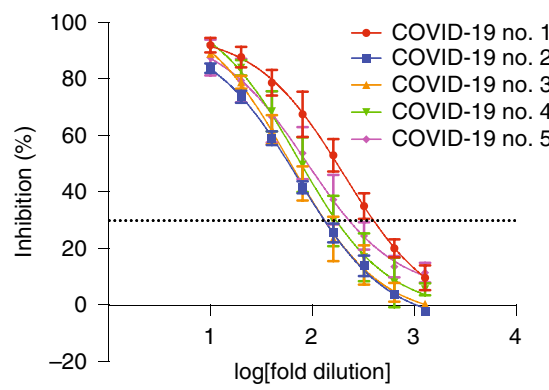

Low IgM/high IgG

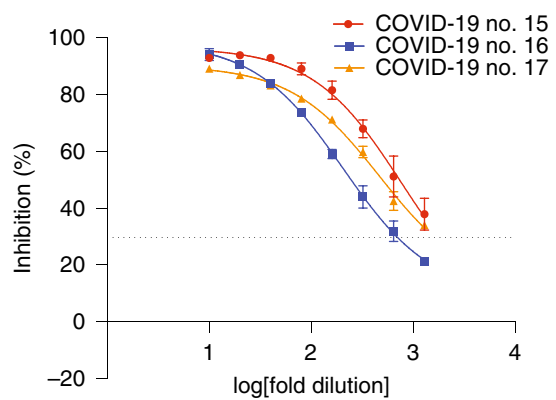

b

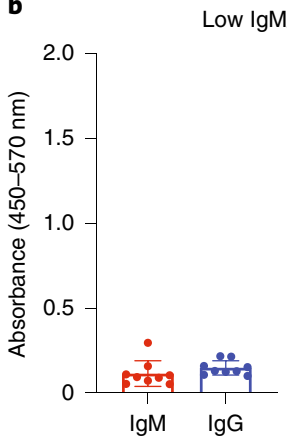

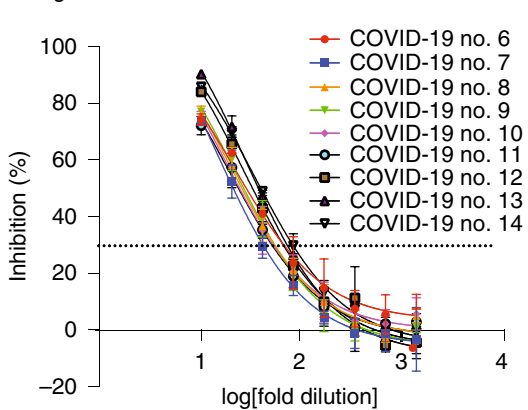

d

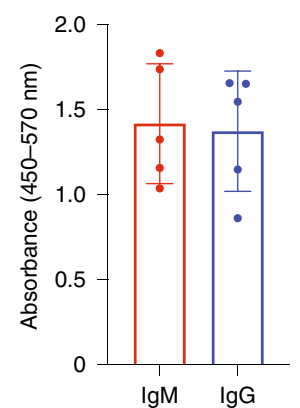

High IgM/high IgG

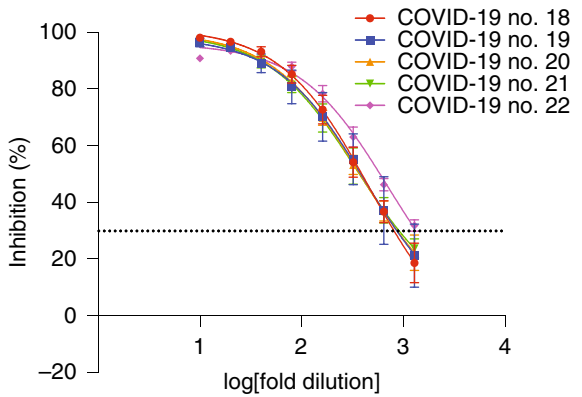

Fig. 2 | Isotype-independent neutralization by human sera with different levels of IgM and IgG antibodies. a-d, Results for the high IgM/low lgG ( $n=5$ ) (a), low $\operatorname{lgM} / \operatorname{low} \operatorname{lgG}(n=9)(\mathbf{b})$, low $\lg M /$ high $\lg G(n=3)$ (c) and high $\operatorname{lgM} /$ high $\lg G(n=5)$ (d) groups. The $\lg M$ and $\lg G$ levels were determined by isotype-specific capture ELISA at 1:50 dilution, as detailed in the Methods. Each data point in the IgM/lgG capture ELISA panel represents a different serum sample from a patient with COVID-19. Inhibition curves of sVNT for each of the sera from patients with COVID-19 are shown on the right. The dotted line indicates the sVNT cutoff at 30\%. The data presented are the mean of two independent experiments. The serum sample numbers are assigned for this study alone and not related to the sample numbers in the 60-serum panel used in Fig. 4. Error bars, s.d.

demonstrating the isotype-independent performance of the assay. It is worth noting that for the panel with low IgM/low IgG, a 70-90\% inhibition is still achieved in sVNT, demonstrating its superior sensitivity, as this group of sera was deemed negative or weakly positive with isotype-specific capture ELISA based on IgM or IgG alone. It is interesting to note that the slope of titer drop was greatest in the low IgM/low IgG group, followed by the high IgM/low IgG group.

We then tested different animal sera in the sVNT assays to demonstrate species-independent performance. Results from mice and rabbits immunized with the SARS-CoV-2 RBD protein demonstrate very potent neutralizing activity in the SARS-CoV-2 sVNT (Fig. 3a). Similarly, sera from ferrets infected with SARS-CoV and rabbits immunized with inactivated SARS-CoV also display an efficient dose-dependent inhibition of the interaction between hACE2 and the SARS-CoV RBD in the SARS-CoV sVNT (Fig. 3b).

Specificity against other human CoVs and comparison of SARS sera collected in 2003 versus 2020. To demonstrate specificity, we tested different panels and confirmed that the SARS-CoV-2 sVNT can differentiate antibody responses to SARS-CoV-2 from those to other human $\mathrm{CoV}$ infections (Fig. 3c). For human sera from patients with 229/NL63 or OC43 infection and alpaca sera from experimental MERS-CoV infection, there is no detectable cross-neutralization. For SARS sera, there is some level of cross-reactivity, not unexpected given their close genetic relatedness and what was reported previously $y^{3,7}$. When analyzed by the SARS-CoV and SARS-CoV-2 sVNT assays side-by-side, neutralizing sera from patients who had SARS could be differentiated from sera from patients who had COVID-19 (Fig. 3d,e).

During the investigation of potential cross-reactivity between SARS sera and SARS-CoV-2 virus, we made several notable observations. First, despite the lack of cross-neutralization by SARS sera against the live SARS-CoV-2 virus in cVNT observed by us and other groups ${ }^{13,14}$, we detected some level of cross-neutralization in sVNT (Fig. 3c), indicating that sVNT is more sensitive than cVNT. Second, SARS NAbs are detectable for at least 17 years in recovered patients (Fig. 3c,e). Third, the cross-neutralization level is higher in SARS sera sampled in 2020 than in those sampled in 2003 (Fig. 3c), although the homologous neutralizing level of the 2020 sera (Fig. 3e) is lower than that of the 2003 sera (Fig. 3d); this is also confirmed by determining RBD-binding antibodies using an indirect ELISA assay (Extended Data Fig. 3). Finally, we have found that the $\mathrm{N}$-specific antibody level is much lower in the 2020 SARS sera than in the 2003 samples (Fig. 3f).

Correlation between biochemical sVNT and live virus cVNT and pVNT. A panel of 60 COVID-19 sera with different levels of SARS-CoV-2 NAbs first determined by sVNT were chosen for a comparative and correlation study between sVNT and two other VNTs, cVNT and pVNT. As shown in Fig. $4 a-c$, all three VNT assays had a good overall correlation, with sVNT-cVNT correlating slightly better than sVNT-pVNT or pVNT-cVNT. The sVNT titer was calculated using the half-maximum inhibitory concentration $\left(\mathrm{IC}_{50}\right.$; Supplementary Table 2). As demonstrated in Extended Data Fig. 2 using mAbs, not all RBD-binding antibodies are NAbs, but RBD remains a suitable antigen to estimate NAb levels in sera from patients with COVID-19. In addition, as shown in Extended Data Fig. 4, there is also a good correlation between the results of the RBD ELISA and the RBD sVNT.

Validation with two cohorts of positive and negative sera from two countries. To validate the performance of the SARS-CoV-2 sVNT, we tested two different cohorts of positive and negative sera. 

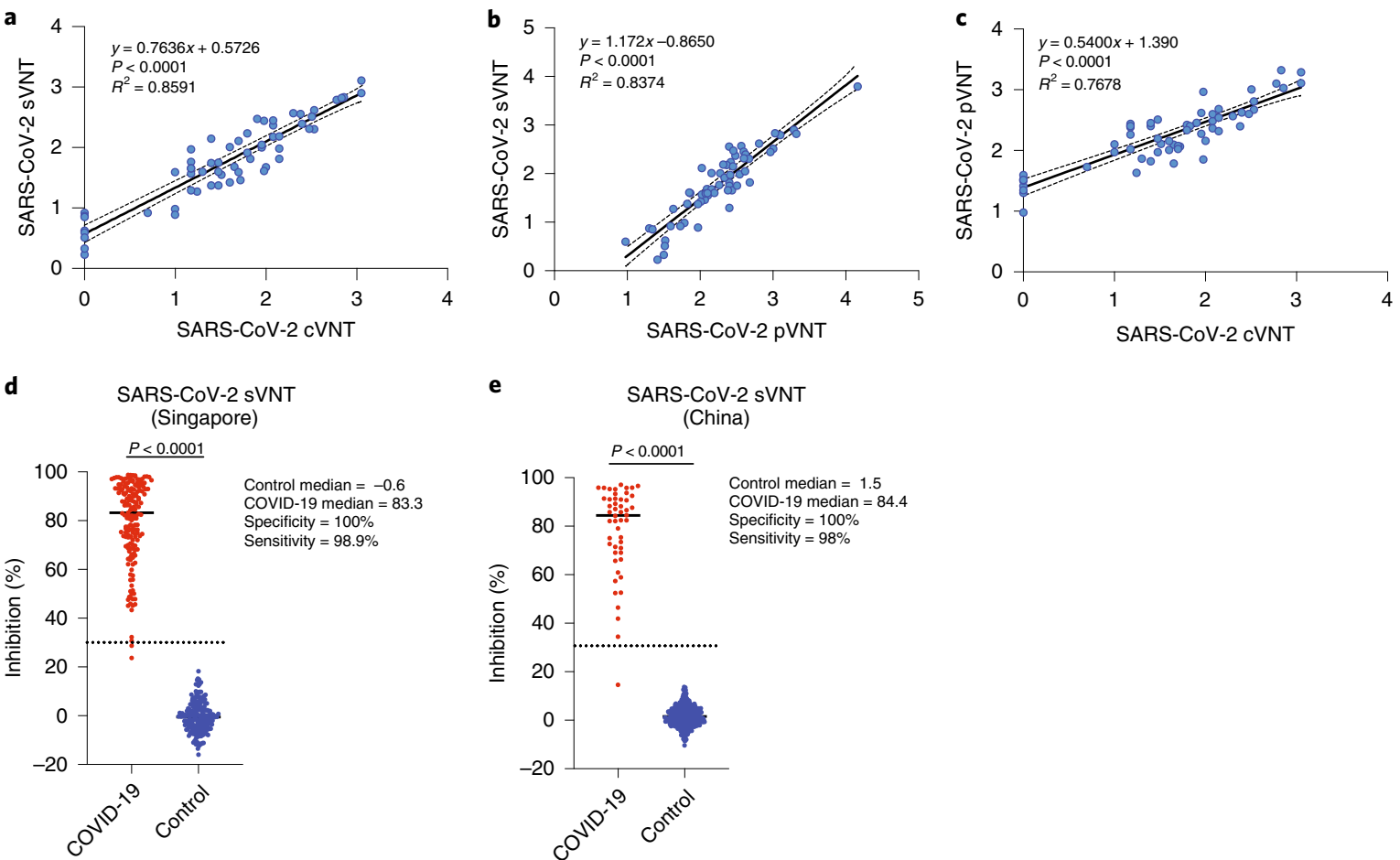

Fig. 4 | Specificity, sensitivity and robustness of sVNT. a-c, Correlation analysis for 60 COVID-19 convalescence sera with different levels of SARS-CoV-2 NAbs by sVNT and cVNT (a), sVNT and pVNT (b), and pVNT and cVNT (c). Correlation and linear regression analyses were performed in GraphPad Prism using Pearson's correlation coefficients. Statistical significance was calculated using the two-tailed test. The data presented are the log of the neutralization titer for $\mathrm{CVNT}$ and the log of the IC $\mathrm{C}_{50}$ value for SVNT or $\mathrm{PVNT}$ and are the mean from two independent experiments. The dashed lines indicate the standard deviations of the linear regression plots. d,e, Testing of healthy control and COVID-19 serum cohorts in Singapore (d) $($ COVID-19 $n=175$, control $n=200)$ and Nanjing, China (COVID-19 $n=50$, control $n=200$ ) (e) at the final serum dilution of 1:20. The horizontal lines indicate the median values. The dotted lines represent the cutoff at $30 \%$ inhibition. The $P$ values presented in $\mathbf{d}$ and $\mathbf{e}$ were calculated from unpaired two-tailed Student's $t$-tests.

The assay was performed in two different countries by two independent groups to further ensure reliability and reproducibility. A cutoff at $30 \%$ inhibition was chosen from testing over 500 negative human sera. For the first cohort, we tested 175 sera from patients with PCR-confirmed COVID-19 in Singapore collected on days 14-33 after symptom onset and 200 healthy control sera, resulting in $100 \%$ specificity and sensitivity of $98.9 \%$ (Fig. 4 d). For the second cohort, we tested 50 sera from patients with PCR-confirmed COVID-19 in Nanjing, China, sampled on days 27-61 after symptom onset and 200 healthy control sera. The specificity is $100 \%$ while sensitivity is $98 \%$ (Fig. 4 e).

\section{Discussion}

We are now seven months into the COVID-19 outbreak and the attention worldwide, both for the scientific community and for policymakers, has shifted focus from acute diagnostic strategy and capacity to the use of serology as an important part of a lockdown exit strategy', relying on accurate assessment of infection prevalence and protective immunity at the individual and population (herd) levels. Discussion and debate on the role of serology have intensified greatly in this context ${ }^{6}$.

Although many COVID-19 laboratory-based or point-of-care antibody test kits are commercially available, none is capable of measuring NAbs. The cVNT and pVNT platforms remain the only platforms for detection of NAbs. However, both require live viruses and cells, highly skilled operators, and days to obtain results. They are thus not suitable for mass production and testing on a commercial scale, even in the most developed nations.

The World Health Organization has recently cautioned that positive results from antibody tests do not equal protective immunity ${ }^{15}$ owing to both technical and scientific challenges. First, most, if not all, testing currently performed at the large scale is for detection of BAbs alone and does not measure true NAbs; second, the presence of NAbs may or may not correlate with protection. Although the latter challenge will take much more in-depth scientific and clinical research to resolve in the specific context of COVID-19, past experiences with viral infection in general argue that, in most recovered patients, the NAb level is a good indicator of protective immunity, despite the fact that there are known exceptions to this 'rule of thumb ${ }^{16,17}$. In this study, we have developed a serological platform to tackle the first technical challenge.

The data presented here demonstrated that sVNT is as specific as, but more sensitive than, cVNT in the cell types tested here (Fig. 4). In our initial optimization studies, we found that the RBD protein performed better than the $\mathrm{S} 1$ protein (Extended Data Fig. 1). We have also compared the RBD proteins produced in insect and mammalian cells and found very similar performance (Extended Data Fig. 5). It is still possible to further improve the sensitivity of the sVNT platform in future by protein engineering on either the RBD- or the ACE2-binding interface. The $\mathrm{mAb}$ studies presented in Extended Data Fig. 2 demonstrate that the RBD sVNT measures genuine NAbs, whereas the RBD ELISA is unable to differentiate between BAbs and NAbs. It can therefore be concluded that the RBD-based sVNT is a robust assay platform for reliable quantification of RBD-targeting NAbs. It should be noted that not all NAbs are necessarily RBD-binding antibodies, as indicated by past studies with SARS-CoV that show antibodies to other regions in the S1 or S2 protein can also play a role in virus neutralization ${ }^{18}$. However, studies based on both SARS-CoV and SARS-CoV-2 suggest that the RBD-targeting 

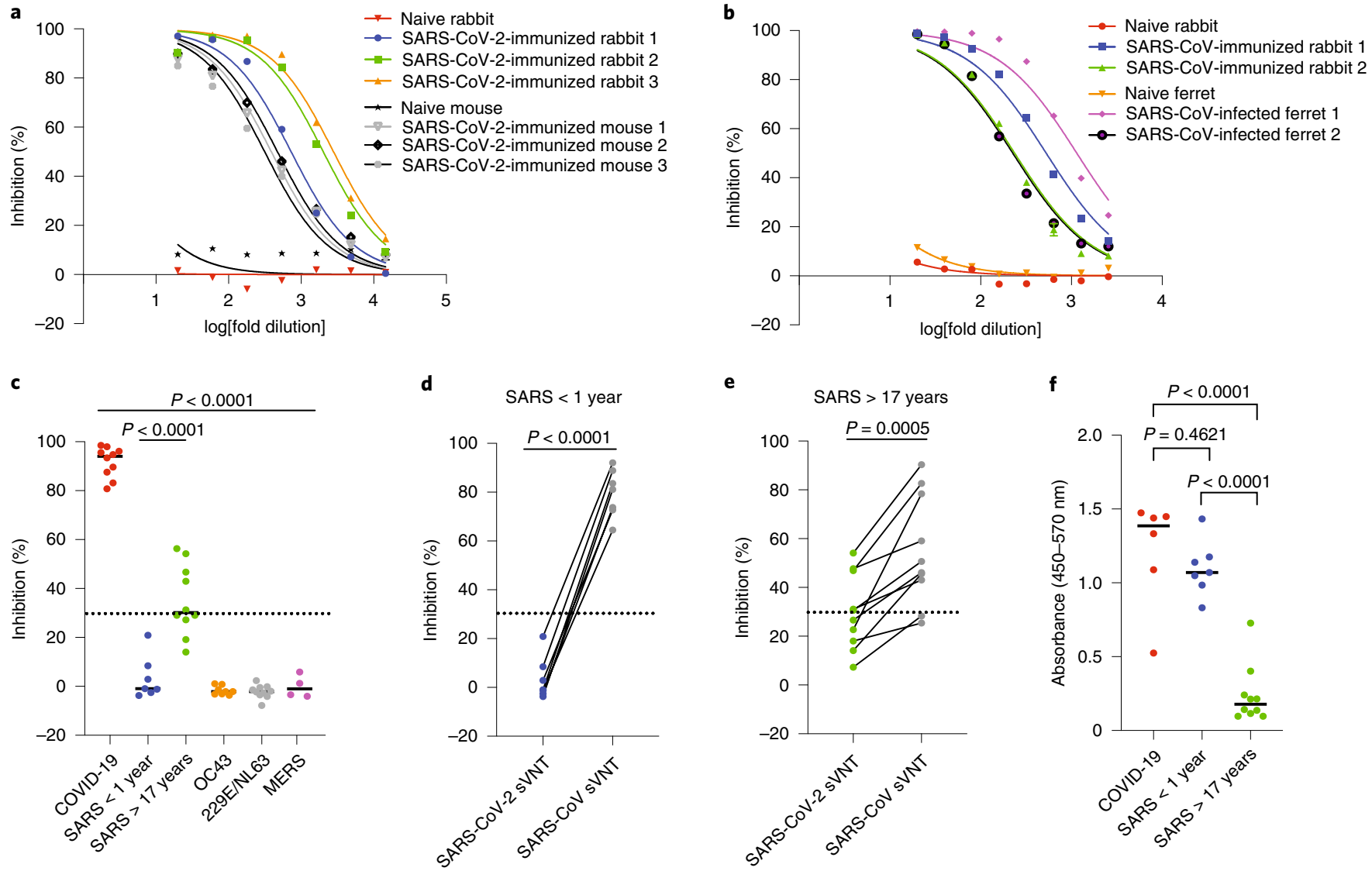

Fig. 3 | Species-independent and virus-specific neutralization. $\mathbf{a}, \mathbf{b}, \mathrm{sVNT}$ analysis of rabbit anti-SARS-CoV-2 RBD sera from immunized rabbits ( $n=3$ ) and mice $(n=3)(\mathbf{a})$; and ferret anti-SARS-CoV sera from infection $(n=2)$ and rabbit anti-SARS-CoV sera from immunization $(n=2)(\mathbf{b})$. The data presented are the mean of two biological replicates. c, The specificity of the SARS-CoV-2 sVNT was determined using different coronavirus sera: human COVID-19 sera $(n=10)$, human SARS sera sampled in 2003 ( $n=7,<1$ year), human SARS-CoV sera sampled in 2020 ( $n=10,>17$ years), human OC43 sera $(n=8)$, human 229E/NL63 sera $(n=10)$, MERS-CoV sera from experimentally infected alpaca $(n=4)$. d,e, Comparative analysis of homologous and heterologous NAb levels for the 2003 SARS (d) and 2020 SARS (e) serum panels using both SARS-CoV-2 sVNT and SARS-CoV sVNT. The dotted lines represent the sVNT cutoff at 30\% inhibition. f, Comparative analysis of homologous N-specific antibodies in the three serum cohorts indicated: SARS-CoV-2 N protein indirect ELISA for COVID-19 sera and SARS-CoV N protein indirect ELISA for the two SARS serum panels, respectively. Unpaired and paired two-tailed Student's $t$-tests were used in $\mathbf{c}, \mathbf{f}$ and $\mathbf{d}, \mathbf{e}$, respectively. All statistical analyses were performed using GraphPad Prism. The horizontal lines indicate the median values.

NAbs are immunodominant during both SARS and COVID-19 infections ${ }^{19,20}$. In our study, we used 60 patient serum samples of varying NAb levels, and the 3-way correlation studies presented in Fig. 4 clearly demonstrate that the correlation between sVNT and cVNT is as good as, if not better than, that between pVNT and cVNT. This indicates that non-RBD-targeting antibodies, which could be measured in pVNT, but not in sVNT, are unlikely to play a major role in SARS-CoV-2 neutralization, consistent with previous findings ${ }^{19-21}$.

The major advantage of sVNT is that it can be rapidly conducted in most research or clinical laboratories without the need to use live biological materials and biosafety containment. The sVNT is also amenable to high-throughput testing and/or fully automated testing after minimal adaptation.

Another advantage of sVNT is its ability to detect SARS-CoV-2 antibodies in a species-independent manner. As the origin of SARS-CoV-2 and early transmission events remain elusive, the sVNT assay will be ideally suited for 'virus hunting', as past studies have amply demonstrated that serological surveys are more superior than molecular detection considering that virus-specific antibodies last much longer than viral genetic material in animals ${ }^{22-24}$. Sampling serum for antibody detection is also more reliable than other sampling approaches used for molecular detection, as the target tissues can vary from virus to virus ${ }^{25-27}$.

In addition, sVNT offers a key advantage over most ELISA or point-of-care tests in its ability to detect total NAbs in an isotype-independent manner. This will not only simplify the testing strategy but also further increase the test sensitivity. As shown in Fig. $2 \mathrm{~b}$ for the serum panel of patients with COVID-19 showing low IgM and IgG in the isotype-specific ELISAs, the sVNT assay still detected a substantial level of NAbs. Although the mechanism needs further investigation, there are at least two possibilities: the presence of other immunoglobulin isotypes or neutralization synergy (cooperativity from the combination of different isotype antibodies targeting different neutralization-critical epitopes), as previously observed for HIV and other viruses ${ }^{28-30}$. Our preliminary analyses indicate that neutralization synergy is more likely the mechanism. First, from the human mAb study, we have found some evidence of synergy between two neutralizing mAbs, AR6949 and AR6959 (Extended Data Fig. 2i). Second, IgA testing indicates that there was no high level of RBD-specific IgA in the low IgM/low IgG group (Extended Data Fig. 6).

The results obtained from the two SARS serum panels of 17 years apart are notable. The presence of long-lasting NAbs 17 years 
after the initial infection is encouraging news for patients who have recovered from COVID-19, considering the close relationship between the two viruses. The mechanism and biological significance of the increased cross-neutralization towards SARS-CoV-2 coupled with the decrease/disappearance of $\mathrm{N}$-specific antibodies 17 years after infection warrant further investigation in the context of better understanding SARSr-CoV immune response dynamics.

In summary, we have addressed the challenge of COVID-19 serology with an approach that enables the detection of NAbs in an easy, safe and rapid manner with enhanced specificity and sensitivity. Although the sVNT assay may never be able to completely replace cVNT, our data indicate that the performance of sVNT is well correlated with that of both cVNT and pVNT. Its application can cover many aspects of COVID-19 investigation from contact tracing, seroprevalence surveying and reservoir/intermediate animal tracking to the assessment of herd immunity and longevity of protective immunity. It can also be used to assess vaccine efficacy during preclinical and clinical trials of different vaccine candidates and to monitor neutralizing titers in vaccinees after mass vaccination in human populations.

\section{Online content}

Any methods, additional references, Nature Research reporting summaries, source data, extended data, supplementary information, acknowledgements, peer review information; details of author contributions and competing interests; and statements of data and code availability are available at https://doi.org/10.1038/ s41587-020-0631-z.

Received: 14 June 2020; Accepted: 8 July 2020;

Published online: 23 July 2020

\section{References}

1. Wang, C., Horby, P. W., Hayden, F. G. \& Gao, G. F. A novel coronavirus outbreak of global health concern. Lancet 395, 470-473 (2020).

2. COVID-19 Status Report (WHO, accessed 1 July 2020); https://www.who.int/ emergencies/diseases/novel-coronavirus-2019/situation-reports

3. Zhou, P. et al. A pneumonia outbreak associated with a new coronavirus of probable bat origin. Nature 579, 270-273 (2020).

4. Coronaviridae Study Group of the International Committee on Taxonomy of Viruses The species Severe acute respiratory syndrome-related coronavirus: classifying 2019-nCoV and naming it SARS-CoV-2. Nat. Microbiol. 5 536-544 (2020).

5. Peiris, J. S. et al. Coronavirus as a possible cause of severe acute respiratory syndrome. Lancet 361, 1319-1325 (2003).

6. Petherick, A. Developing antibody tests for SARS-CoV-2. Lancet 395, 1101-1102 (2020).

7. Hoffmann, M. et al. SARS-CoV-2 cell entry depends on ACE2 and TMPRSS2 and is blocked by a clinically proven protease inhibitor. Cell 181, 271-280 (2020).

8. Nie, J. et al. Establishment and validation of a pseudovirus neutralization assay for SARS-CoV-2. Emerg. Microbes Infect. 9, 680-686 (2020).
9. Tang, Y. W., Schmitz, J. E., Persing, D. H. \& Stratton, C. W. The laboratory diagnosis of COVID-19 infection: current issues and challenges. J. Clin. Microbiol. 58, e00512-20 (2020).

10. Okba, N. M. A. et al. Severe acute respiratory syndrome coronavirus 2 -specific antibody responses in coronavirus disease patients. Emerg. Infect. Dis. 26, 1478-1488 (2020).

11. Li, W. et al. Angiotensin-converting enzyme 2 is a functional receptor for the SARS coronavirus. Nature 426, 450-454 (2003).

12. Amanat, F. et al. A serological assay to detect SARS-CoV-2 seroconversion in humans. Nat. Med. https://doi.org/10.1038/s41591-020-0913-5 (2020).

13. Ou, X. et al. Characterization of spike glycoprotein of SARS-CoV-2 on virus entry and its immune cross-reactivity with SARS-CoV. Nat. Commun. 11, 1620 (2020).

14. Anderson, D. E. et al. Lack of cross-neutralization by SARS patient sera towards SARS-CoV-2. Emerg. Microbes Infect. 9, 900-902 (2020).

15. Coronavirus: double warning over antibody tests. $B B C$ https://www.bbc.com/ news/uk-52335210 (accessed 18 April 2020).

16. Burton, D. R. Antibodies, viruses and vaccines. Nat. Rev. Immunol. 2, 706-713 (2002)

17. Klasse, P. J. Neutralization of virus infectivity by antibodies: old problems in new perspectives. Adv. Biol. https://doi.org/10.1155/2014/157895 (2014).

18. Jiang, S., Hillyer, C. \& Du, L. Neutralizing antibodies against SARS-CoV-2 and other human coronaviruses. Trends Immunol. 41, 355-359 (2020).

19. Zeng, F. et al. Quantitative comparison of the efficiency of antibodies against S1 and S2 subunit of SARS coronavirus spike protein in virus neutralization and blocking of receptor binding: implications for the functional roles of S2 subunit. FEBS Lett. 580, 5612-5620 (2006).

20. Premkumar, L. et al. The receptor binding domain of the viral spike protein is an immunodominant and highly specific target of antibodies in SARS-CoV-2 patients. Sci. Immunol. https://doi.org/10.1126/sciimmunol.abc8413 (2020).

21. Robbiani, D. F. et al. Convergent antibody responses to SARS-CoV-2 in convalescent individuals. Nature https://doi.org/10.1038/s41586-020-2456-9 (2020).

22. Li, W. et al. Bats are natural reservoirs of SARS-like coronaviruses. Science 310, 676-679 (2005)

23. Young, P. L. et al. Serologic evidence for the presence in Pteropus bats of a paramyxovirus related to equine morbillivirus. Emerg. Infect. Dis. 2, 239-240 (1996).

24. Yob, J. M. et al. Nipah virus infection in bats (order Chiroptera) in peninsular Malaysia. Emerg. Infect. Dis. 7, 439-441 (2001).

25. Smith, I. et al. Identifying Hendra virus diversity in pteropid bats. PLoS ONE 6, e25275 (2011).

26. Barr, J. et al. Isolation of multiple novel paramyxoviruses from pteropid bat urine. J. Gen. Virol. 96, 24-29 (2015).

27. Leroy, E. M. et al. Fruit bats as reservoirs of Ebola virus. Nature 438, 575-576 (2005).

28. Zwick, M. B. et al. Neutralization synergy of human immunodeficiency virus type 1 primary isolates by cocktails of broadly neutralizing antibodies. J. Virol. 75, 12198-12208 (2001).

29. Howell, K. A. et al. Cooperativity enables non-neutralizing antibodies to neutralize ebolavirus. Cell Rep. 19, 413-424 (2017).

30. Besselaar, T. G. \& Blackburn, N. K. The synergistic neutralization of Rift Valley fever virus by monoclonal antibodies to the envelope glycoproteins. Arch. Virol. 125, 239-250 (1992).

Publisher's note Springer Nature remains neutral with regard to jurisdictional claims in published maps and institutional affiliations.

(c) The Author(s), under exclusive licence to Springer Nature America, Inc. 2020 


\section{Methods}

Cells and viruses. Human embryonic kidney (HEK293T) cells (ATCC no. CRL-3216) and African green monkey kidney clone E6 (Vero-E6) cells (ATCC no. CRL-1586) were maintained in Dulbecco's modified Eagle medium (DMEM) supplemented with $10 \%$ fetal bovine serum. SARS-CoV-2, isolate BetaCoV/ Singapore/2/2020 (GISAID accession code EPI_ISL_406973), was used for the VNT on Vero-E6 cells ${ }^{31}$. HEK293T/SARS-CoV-2 spike expression cells were generated by transduction of PQCXIH-SARS-CoV-2 spike, followed by hygromycin selection at a final concentration of $100 \mu \mathrm{g} \mathrm{ml}^{-1}$. SARS-CoV-2 spike pseudotyped virus was generated by infection of HEK293T-SARS-CoV-2 spike cells with vesicular stomatitis virus $\Delta$ G-luc seed virus at a multiplicity of infection of 5 , and collected at $16 \mathrm{~h}$ post-infection.

Plasmids and recombinant proteins. The SARS-CoV-2 N and S and SARS-CoV $\mathrm{N}$ genes were synthesized by GenScript and BioBasic. The SARS-CoV-2 N and SARS-CoV N genes were cloned into the pcDNA3.1 and pDualGC expression vectors according to the manufacturer's instructions. The SARS-CoV- $2 \mathrm{~S}$ gene was cloned into the PQCXIH vector. The hACE2 gene was amplified from the pCAGGS-hACE2 plasmid (gift from Z. Shi, Wuhan Institute of Virology, China) and subcloned into the pQCXIH expression vector. The recombinant SARS-CoV-2 RBD and S1 and SARS-CoV RBD proteins were produced by GenScript using the baculovirus-insect cell expression system. For performance comparison, recombinant SARS-CoV-2 RBD was also produced by GenScript in the mammalian expression system. The sequence information for these recombinant proteins is shown in Supplementary Table 3. Protein purity was determined by SDS-PAGE and the protein concentration was measured by a Nanodrop 2000. HRP conjugation was performed by adding activated HRP to RBD in 2:1 mass ratio, followed by incubation for $2 \mathrm{~h}$ in the dark at $25 \pm 2{ }^{\circ} \mathrm{C}$ with constant shaking. Sodium borohydride was added into the conjugation reaction to a final concentration of $200 \mu \mathrm{g} \mathrm{ml}^{-1}$, followed by incubation for $2 \mathrm{~h}$ in the dark at $25 \pm 2^{\circ} \mathrm{C}$ with constant shaking. The HRP-conjugated proteins were dialyzed in PBS and the purified HRP-conjugated proteins were kept in $10 \mathrm{mg} \mathrm{ml}^{-1} \mathrm{BSA}$ and preserved in $0.01 \%$ thimerosal. SARS-CoV-2 and SARS-CoV polyhistidine-tagged $\mathrm{N}$ proteins were expressed from the pcDNA3.1 SARS-CoV-2 N and pDualGC SARS-CoV N plasmids, respectively. Expression was conducted in transfected HEK293T cells and the resulting proteins were purified using Ni Sepharose (GE Healthcare) following the manufacturer's instructions. In brief, $20 \mu \mathrm{g}$ pcDNA3.1 SARS-CoV-2 $\mathrm{N}$ or pDualGC SARS-CoV N was used to transfect HEK293T cells. At $48 \mathrm{~h}$ post-transfection, the cells were lysed with cell lysis buffer $(20 \mathrm{mM}$ Tris, $300 \mathrm{mM}$ $\mathrm{NaCl}, 1 \%$ Triton X-100, $0.1 \%$ SDS, $10 \mathrm{mM} \beta$-mercaptoethanol, $25 \mathrm{mM}$ imidazole, $\mathrm{pH}$ 8.0). Clarified cell lysate was pre-incubated with Ni Sepharose overnight at $4^{\circ} \mathrm{C}$ with constant rotation. Polyhistidine-tagged $\mathrm{N}$ proteins were eluted from the $\mathrm{Ni}$ Sepharose with gradient imidazole buffer $(20 \mathrm{mM}$ Tris, $300 \mathrm{mM} \mathrm{NaCl}, 50-500 \mathrm{mM}$ imidazole, $\mathrm{pH}$ 8.0). Fractions containing purified protein were pooled and dialyzed against $20 \mathrm{mM}$ Tris, $300 \mathrm{mM} \mathrm{NaCl}$, pH 8.0. Purified protein concentration was determined by a Nanodrop instrument.

Panels of human and animal sera used in this study. In Singapore, the sera from patients with COVID-19 used in this study were from the Singapore PROTECT study as described previously ${ }^{31}$. Sera from patients who had recovered from SARS from 2003 were as previously described ${ }^{32}$. For SARS recall sampling in 2020, we contacted and obtained blood from consenting individuals previously admitted for SARS (ethics approval number: NHG DSRB E 2020/00091). The human CoV serum panel included post-infection samples from subjects confirmed positive for CoV 229/NL63 and CoV OC43 using the SeeGene RV12 respiratory multiplex kit in a previous study (ethics approval number: NUS-IRB 11-3640) ) $^{32}$. Negative control sera were obtained from residual serum samples from previous unrelated studies. In Nanjing, China, sera from convalescent patients with COVID-19 were collected with written informed consent and approved by the ethics committee of The Second Hospital of Nanjing (ethics approval number: 2020-LS-ky003). Mouse and rabbit anti-SARS-CoV-2 RBD sera and monoclonal antibodies raised against the SARS-CoV-2 RBD were all from GenScript. Rabbit and ferret anti-SARS-CoV sera and alpaca anti-MERS-CoV sera were as described in previous studies ${ }^{33,34}$.

Direct binding and sVNT assay. A MaxiSORP ELISA plate (Nunc) was pre-coated with hACE2 protein (GenScript) at $100 \mathrm{ng}$ per well in $50 \mu \mathrm{l}$ of $100 \mathrm{mM}$ carbonatebicarbonate coating buffer ( $\mathrm{pH} 9.6$ ) overnight at $4{ }^{\circ} \mathrm{C}$, followed by blocking with OptEIA assay diluent (BD). For the direct binding assay, HRP-conjugated SARS-CoV-2 N, S1 or RBD or HRP-conjugated SARS-CoV RBD (all produced by GenScript; Supplementary Table 3) was added to the hACE2-coated plate at different concentrations in $100 \mu \mathrm{l}$ of OptEIA assay diluent (BD) for $1 \mathrm{~h}$ at room temperature. Unbound HRP-conjugated antigens were removed by five washes with phosphate-buffered saline, $0.05 \%$ Tween-20 (PBST). A colorimetric signal was developed on the enzymatic reaction of HRP with a chromogenic substrate, 3,3',5,5'-tetramethylbenzidine (TMB) (Invitrogen). An equal volume of TMB stop solution (KPL) was added to stop the reaction, and the absorbance readings at $450 \mathrm{~nm}$ and $570 \mathrm{~nm}$ were acquired using a Cytation 5 microplate reader (BioTek). For the sVNT assay, $3 \mathrm{ng}$ of HRP-RBD (from either virus) was pre-incubated with test serum for $1 \mathrm{~h}$ at $37^{\circ} \mathrm{C}$ (final volume of $50 \mu \mathrm{l}$ ), followed by addition into a
MaxiSORP ELISA plate coated with hACE2 (100 ng per well, as described above) for $1 \mathrm{~h}$ at room temperature. Unbound HRP-conjugated antigens were removed by five PBST washes. Inhibition $(\%)=(1-$ sample optical density value/negative control optical density value $) \times 100$. For determination of neutralization titers, human sera were used with a twofold serial dilution starting at 1:10, the same as for cVNT and pVNT described below. For positive and negative serum validation in the cohorts from Singapore and China, a final 1:20 dilution of the test serum was used.

ELISA. For the indirect ELISA, $100 \mathrm{ng}$ of each protein was coated onto MaxiSORP plates (Nunc) using $100 \mathrm{mM}$ carbonate buffer and blocked with OptEIA assay diluent (BD). Sera from patients with COVID-19 or SARS were tested at a dilution of 1:50 and detected by goat anti-human IgG-HRP (Santa Cruz) at 1:10,000 dilution. For the capture ELISA, MaxiSORP plates (Nunc) were coated with $10 \mu \mathrm{g} \mathrm{ml}^{-1}$ of anti-human IgM (SeraCare), anti-human IgG (Jackson labs) or anti-human IgA (GenScript) in bicarbonate buffer overnight at $4^{\circ} \mathrm{C}$. Wells were blocked using BD OptEIA assay diluent (BD) for $1 \mathrm{~h}$ at $37^{\circ} \mathrm{C}$ and heat-inactivated sera at 1:50 dilution were added and incubated for $1 \mathrm{~h}$ at $37^{\circ} \mathrm{C}$. Following extensive washing, SARS-CoV-2 HRP-RBD (GenScript) at $4 \mu \mathrm{g} \mathrm{ml}^{-1}$ was added and incubated for $30 \mathrm{~min}$ at $37^{\circ} \mathrm{C}$. The chromogenic reaction was quantified following the addition of TMB substrate (Invitrogen) and stop solution (KPL SeraCare). The absorbance of the samples was measured at $450 \mathrm{~nm}$ and the background at $570 \mathrm{~nm}$.

cVNT and pVNT. For cVNT, $50 \mu \mathrm{l}$ of twofold serial-diluted serum was pre-incubated with $50 \mu \mathrm{l}$ of $1,000 \mathrm{TCID}_{50}$ per milliliter of SARS-CoV-2 in $5 \%$ FBS in DMEM for $90 \mathrm{~min}$ at $37^{\circ} \mathrm{C}$. The virus-serum mixtures were then added into monolayer Vero-E6 cells for $1 \mathrm{~h}$ at $37^{\circ} \mathrm{C}$. At $1 \mathrm{~h}$ post-infection, the inoculum was removed and infected cells were washed once with 5\% FBS in DMEM. Cells were then replenished with 5\% FBS in DMEM and the neutralization titers were determined at 4 dpi. For pVNT, $1.5 \times 10^{6}$ RLU of SARS-CoV- 2 spike pseudotyped virus was pre-incubated with twofold serial-diluted test serum in a final volume of $50 \mu \mathrm{l}$ for $1 \mathrm{~h}$ at $37^{\circ} \mathrm{C}$, followed by infection of ACE2-transfected HEK293T cells. At $18-20 \mathrm{~h}$ post-infection, an equal volume of ONE-Glo luciferase substrate (Promega) was added and the luminescence signal was measured using a Cytation 5 microplate reader (BioTek) with Gen5 software (Version 3.03.14). The same dilution range from 1:20 to 1:1,280 was used to facilitate side-by-side comparison in the correlation studies of the three different VNT assays.

Statistical analysis. Statistical analysis was performed using GraphPad Prism 7 software. The differences between negative control and COVID-19 test sera were analyzed using an unpaired $t$-test. The differences between paired SARS serum in SARS-CoV-2 sVNT and SARS-CoV sVNT were analyzed using a paired $t$-test. Correlations between sVNT and cVNT or pVNT were analyzed using Pearson correlation coefficients. All data presented are derived from two independent experiments.

Reporting Summary. Further information on research design is available in the Nature Research Reporting Summary linked to this article.

\section{Data availability}

The key datasets used in this study are presented in Supplementary Tables 1-3. Additional datasets generated and/or analyzed during the current study are available from the corresponding author on reasonable request.

\section{References}

31. Young, B. E. et al. Epidemiologic features and clinical course of patients infected with SARS-CoV-2 in Singapore. J. Am. Med. Assoc. 323, 1488-1494 (2020).

32. Jiang, L. et al. Detection of viral respiratory pathogens in mild and severe acute respiratory infections in Singapore. Sci. Rep. 7, 42963 (2017).

33. Yu, M. et al. Determination and application of immunodominant regions of SARS coronavirus spike and nucleocapsid proteins recognized by sera from different animal species. J. Immunol. Methods 331, 1-12 (2008).

34. Crameri, G. et al. Experimental infection and response to rechallenge of alpacas with Middle East respiratory syndrome coronavirus. Emerg. Infect. Dis. 22, 1071-1074 (2016).

\section{Acknowledgements}

We thank S. Tang, Y. Shen, N. Mao, W. Shao and L. Zhu for technical assistance, advice and logistics support with assay development and testing; Y. Peng, A. Gamage, B. L. Lim and X. M. Ong for assistance with protein purification, sample management and testing; Y. Abdad and L. W. L. Tan for help with human CoV serum collection; V. Vijayan, B. Ng and V. Sivalingam of the Duke-NUS Medical School ABSL3 facility for logistics management and assistance. L.-F.W. and D.E.A. are supported by grants from the Singapore National Research Foundation (NRF2016NRF-NSFC002-013) and the National Medical Research Council (STPRG-FY19-001 and COVID19RF-003).

\section{Author contributions}

L.-F.W. conceived and guided the study. C.W.T., W.N.C., X.Q., P.L., C.T., V.C.-W.C., W.R.S., R.F. and D.E.A. performed laboratory work including data analysis. M.I.-C.C., 
Z.H., B.E.Y., Y.-J.T., Y.Y. and D.C.L. provided necessary samples and coordination for the study. L.-F.W. initiated the manuscript writing with input from all authors.

\section{Competing interests}

A patent application has been filed for the content disclosed in this study and a

SARS-CoV-2 sVNT kit is in the process of commercialization with industrial partners.

\section{Additional information}

Extended data is available for this paper at https://doi.org/10.1038/s41587-020-0631-z. Supplementary information is available for this paper at https://doi.org/10.1038/ s41587-020-0631-z.

Correspondence and requests for materials should be addressed to D.E.A. or L.-F.W. Reprints and permissions information is available at www.nature.com/reprints. 


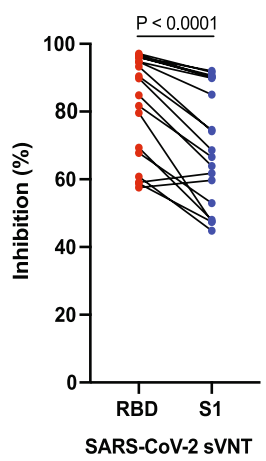

Extended Data Fig. 1 | Comparison of SARS-CoV-2 RBD and S1 sVNT. Equal molar ratio of HRP-conjugated SARS-CoV-2 RBD or S1 was used to detect NAbs from 20 test sera at a 1:20 dilution. Both recombinant RBD and S1 proteins were produced from the baculovirus-insect cell expression system. Statistical analysis was performed using paired two-tailed Student's t-test. 

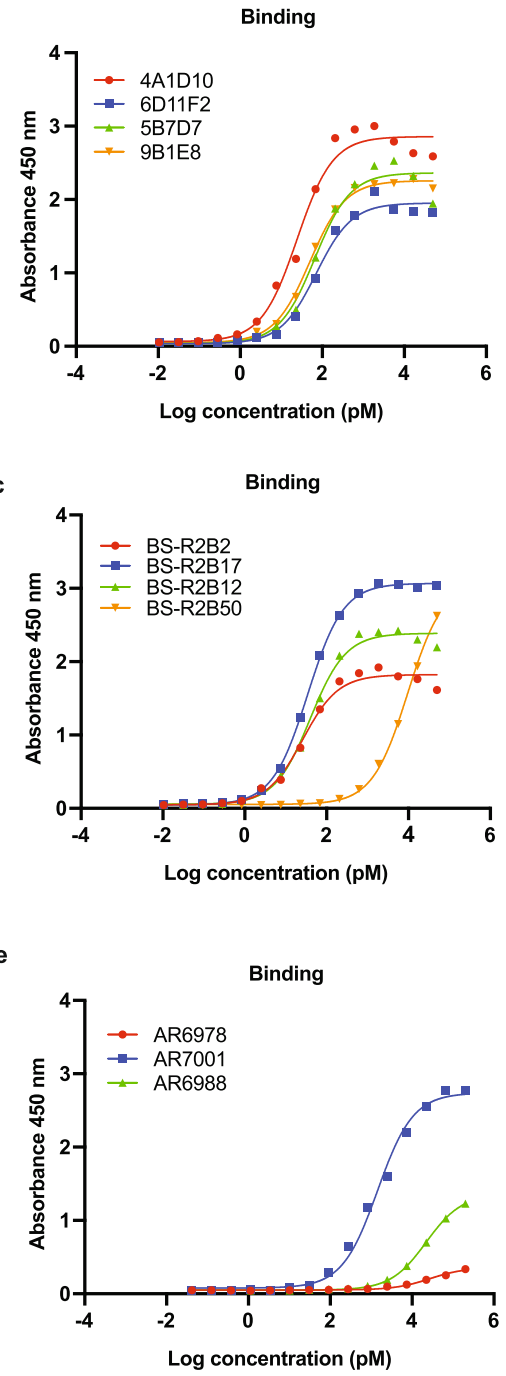

g

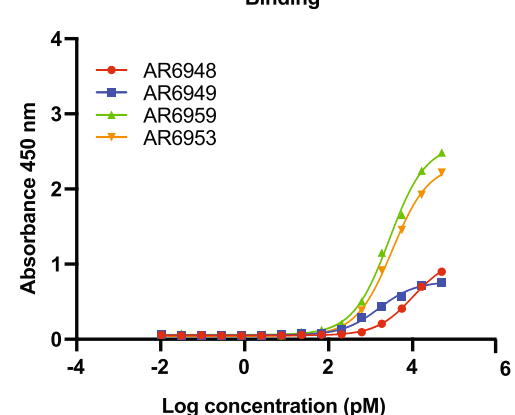

b

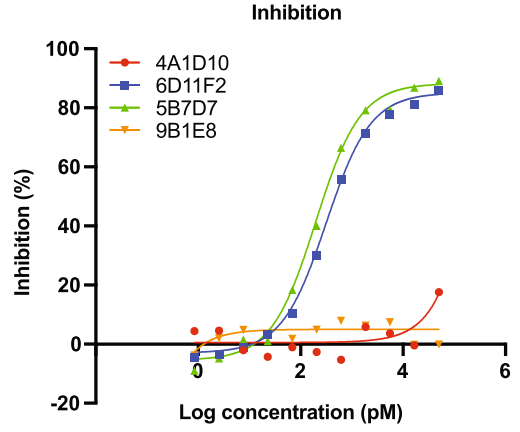

d
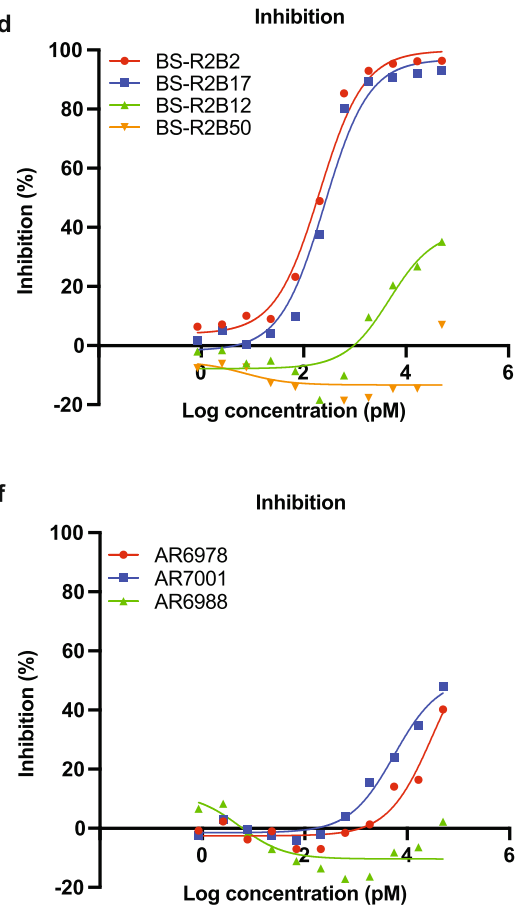

h

Inhibition

Inhibition

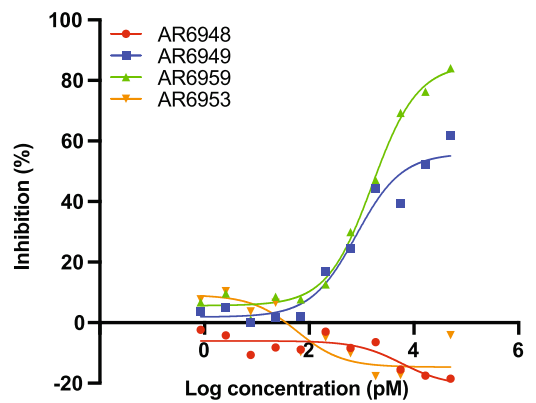

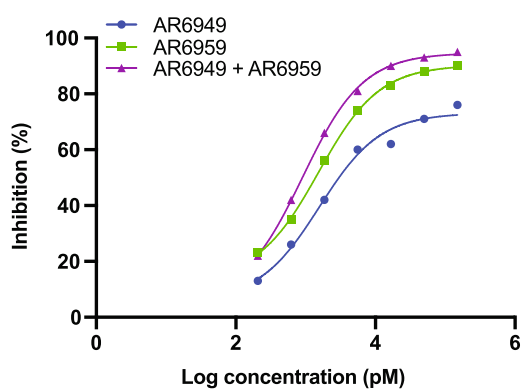

Extended Data Fig. 2 | Comparative analysis of SARS-CoV-2 sVNT and indirect RBD ELISA with monoclonal antibodies. The species origin of monoclonal antibodies are as follows: 4 from mouse $(\mathbf{a}, \mathbf{b}) ; 4$ from rabbit $(\mathbf{c}, \mathbf{d}) ; 3$ from llama (e, f); and 4 from human ( $\mathbf{g}$, $\mathbf{h})$. For each set of sera, indirect RBD-binding ELISA ( $\mathbf{a}, \mathbf{c}, \mathbf{e}, \mathbf{g})$ and RBD-blocking sVNT ( $\mathbf{b}, \mathbf{d}, \mathbf{f}, \mathbf{g}$ ) were conducted side-by-side. For the two human NAbs, a synergy comparative study was also conducted (i). 
a

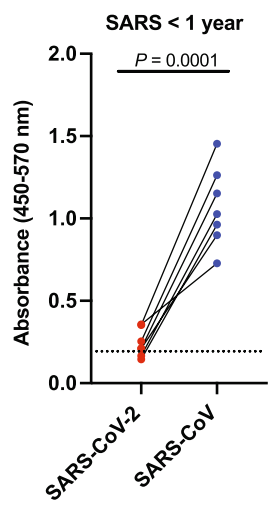

b

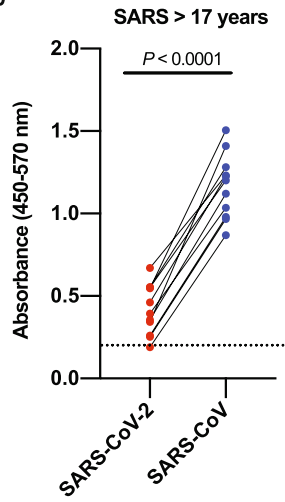

Extended Data Fig. 3 | Comparative ELISA analysis of SARS sera sampled collected in 2003 and 2020 against RBD of SARS-CoV-2 and SARS-CoV. Testing was conducted using SARS patient serum collected $(\mathbf{a})<1$ year of infection $(n=7)$, and $(\mathbf{b})>17$ years post infection $(n=10)$. Indirect ELISA was carried out as described in Methods with patient sera used at the final dilution of 1:50. Paired two-tailed Student's t-test was used for statistical significance analysis. 


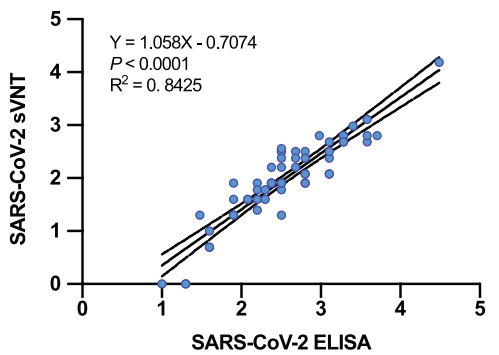

Extended Data Fig. 4 | Correlation of SARS-CoV-2 sVNT and indirect RBD ELISA. Pearson's correlation coefficient and linear regression analysis was performed using end-point titer of SARS-CoV-2 sVNT and ELISA using the same 60-serum panel as that in Fig. 4. Dashed line indicates the standard deviation from the linear regression analysis. Statistical significance was determined using the two-tailed test. 


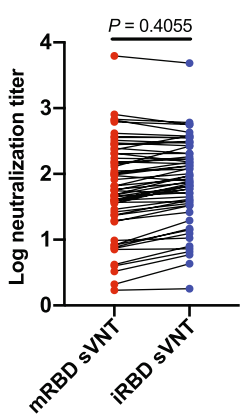

b

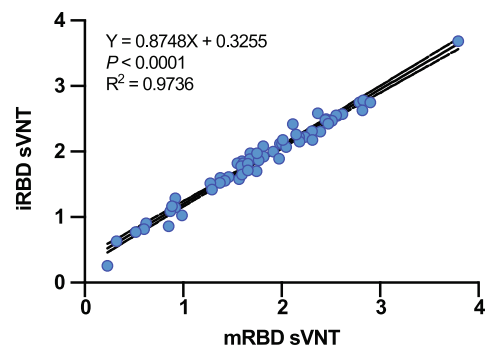

Extended Data Fig. 5 | Performance correlation of sVNTs using two different recombinant RBD proteins. Using the same 60-serum panel as that in Fig. 4, the sVNT performance based on the insect RBD (iRBD) and the mammalian RBD (mRBD) was compared by (a) neutralization activities in log IC 50 value or by (b) Two-tailed Pearson's correlation and linear regression analyses of the log $I C_{50}$ values from panel a. Paired two-tailed Student's t-test was used in panel a. 
ARTICLES

NATURE BIOTECHNOLOGY
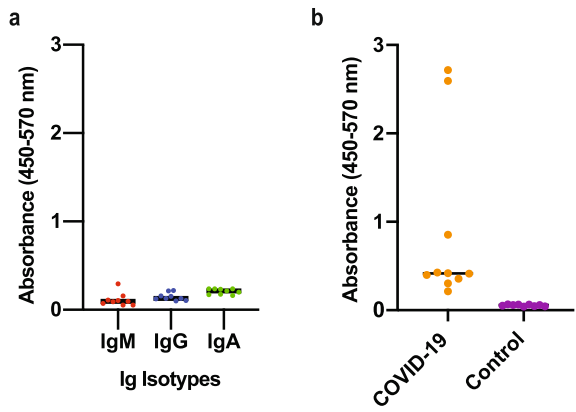

Extended Data Fig. 6 | Determination of serum IgA levels using capture ELISA. A comparative analysis was conducted using (a) the serum panel with low IgN and low lG from panel Fig. Lb $(n=9) ;(b)$ selective COVID-19 patient serb with known high level of lg $(n=2)$ used as positive control; and (c) negative control serra $(n=10)$.

NATURE BIOTECHNOLOGY | www.nature.com/naturebiotechnology 


\section{Reporting Summary}

Nature Research wishes to improve the reproducibility of the work that we publish. This form provides structure for consistency and transparency in reporting. For further information on Nature Research policies, see our Editorial Policies and the Editorial Policy Checklist.

\section{Statistics}

For all statistical analyses, confirm that the following items are present in the figure legend, table legend, main text, or Methods section.

$\mathrm{n} / \mathrm{a}$ Confirmed

$\bigotimes$ The exact sample size $(n)$ for each experimental group/condition, given as a discrete number and unit of measurement

$\bigotimes$ A statement on whether measurements were taken from distinct samples or whether the same sample was measured repeatedly

$\triangle$ The statistical test(s) used AND whether they are one- or two-sided

Only common tests should be described solely by name; describe more complex techniques in the Methods section.

Х $\square$ A description of all covariates tested

Х $\square$ A description of any assumptions or corrections, such as tests of normality and adjustment for multiple comparisons

$\triangle$ A full description of the statistical parameters including central tendency (e.g. means) or other basic estimates (e.g. regression coefficient)

AND variation (e.g. standard deviation) or associated estimates of uncertainty (e.g. confidence intervals)

$\triangle$ For null hypothesis testing, the test statistic (e.g. $F, t, r$ ) with confidence intervals, effect sizes, degrees of freedom and $P$ value noted

Give $P$ values as exact values whenever suitable.

Х $\square$ For Bayesian analysis, information on the choice of priors and Markov chain Monte Carlo settings

Х $\square$ For hierarchical and complex designs, identification of the appropriate level for tests and full reporting of outcomes

$\square \bigotimes$ Estimates of effect sizes (e.g. Cohen's $d$, Pearson's $r$ ), indicating how they were calculated

Our web collection on statistics for biologists contains articles on many of the points above.

\section{Software and code}

Policy information about availability of computer code

Data collection Gen5 software version 3.03.14

Data analysis GraphPad Prism 7

For manuscripts utilizing custom algorithms or software that are central to the research but not yet described in published literature, software must be made available to editors and

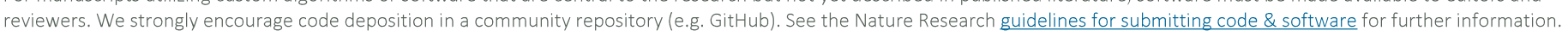

\section{Data}

Policy information about availability of data

All manuscripts must include a data availability statement. This statement should provide the following information, where applicable:

- Accession codes, unique identifiers, or web links for publicly available datasets

- A list of figures that have associated raw data

- A description of any restrictions on data availability

Key datasets used in this study are presented in Supplementary tables. Additional datasets generated and/or analyzed during the current study are available from the corresponding author on reasonable request. 
Please select the one below that is the best fit for your research. If you are not sure, read the appropriate sections before making your selection.

$\bigotimes$ Life sciences $\quad \square$ Behavioural \& social sciences $\quad \square$ Ecological, evolutionary \& environmental sciences

For a reference copy of the document with all sections, see nature.com/documents/nr-reporting-summary-flat.pdf

\section{Life sciences study design}

All studies must disclose on these points even when the disclosure is negative. \begin{tabular}{l|l} 
Sample size & $\begin{array}{l}\text { Sample size was determined by the available number of convalescent sera from PCR confirmed patients. Positive and negative control } \\
\text { samples used for initial assay development showed a clear different in reactivity. For the correlation study, a sample size of } 60 \text { well- }\end{array}$
\end{tabular} characterized sera were used and deemed more than sufficient for statistical/correlation analyses.

Data exclusions All data was included in the analysis

Replication Surrogate virus neutralization test, pseudotyped virus neutralization test, live virus neutralization test, ELISA were performed in duplication. All attempts at replication were successful.

Randomization Randomization was not performed since the purpose of this work was assay development.

Blinding Whenever necessary and/or possible, such as for the correlation studies using different virus neutralization tests, experiments were conducted blindly by different team members and at different days.

\section{Reporting for specific materials, systems and methods}

We require information from authors about some types of materials, experimental systems and methods used in many studies. Here, indicate whether each material, system or method listed is relevant to your study. If you are not sure if a list item applies to your research, read the appropriate section before selecting a response.

\begin{tabular}{|c|c|c|}
\hline \multicolumn{2}{|r|}{ Materials \& experimental systems } & Methods \\
\hline $\mathrm{n} / \mathrm{a}$ & Involved in the study & \begin{tabular}{l|l}
$\mathrm{n} / \mathrm{a}$ & Involved in the study
\end{tabular} \\
\hline - & $\bigotimes$ Antibodies & $\bigotimes \square$ ChIP-seq \\
\hline 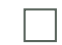 & Eukaryotic cell lines & Х $\square$ Flow cytometry \\
\hline$\bigotimes$ & $\square$ Palaeontology and archaeology & $\bigotimes \mid \square$ MRI-based neuroimaging \\
\hline Х & $\square$ Animals and other organisms & \\
\hline L & $\bigotimes$ Human research participants & \\
\hline Х & $\square$ Clinical data & \\
\hline Х & $\square$ Dual use research of concern & \\
\hline
\end{tabular}

\section{Antibodies}

Antibodies used
1. KPL affinity purified antibody to Human IgM ( $\mu$ ), SeraCare, Catalog number: 5210-0107 (01-10-03), Lot number: 10468165. Dilution used: $10 \mu \mathrm{g} / \mathrm{ml}$.
2. AffiniPure Goat anti-human IgG (H+L), Jackson labs, Catalog number: 109-005-003, Lot number: 147020 . Dilution used: $10 \mu \mathrm{g} / \mathrm{ml}$.
3. Anti-human IgA monoclonal antibody, GenScript, LotT2005015. Dilution used: $10 \mathrm{\mu g} / \mathrm{ml}$.
4. Goat-anti-human IgG-HRP, Santa Cruz Biotechnology, Catalog number: sc-2907, Lot number: J0914. Dilution used: 1:10,000,
5. Mice and rabbits SARS-CoV-2 RBD immunised antisera; anti-SARS-CoV-2 RBD monoclonal antibodies from human (AR6948, AR6949, AR6959, AR6953), mouse (4A1D10, 6D11F2, 5B7D7, 9B1E8), rabbit (BS-R2B2, BS-R2B17, BS-R2B12, BS-R2B50) and llama (AR6978, AR7001, AR6988) were all from GenScript Biotech. Those are in-house produced antibodies which are not commercially available.

Validation

All primary antibodies are from commercial sources and validated by the manufacturers: the specificity of the KPL affinity purified antibody to human $\operatorname{lgM}(\mu)$, AffiniPure Goat anti-human $\lg \mathrm{G}(\mathrm{H}+\mathrm{L})$, and Anti-human $\lg A$ monoclonal antibody were validated by immunoblotting and/or ELISA whereas the SARS-CoV-2 specific antibodies (polyclonal sera or monoclonal antibodies) were validated by SARS-CoV-2 RBD binding ELISA.

\section{Eukaryotic cell lines}

Policy information about cell lines

Cell line source(s)

Vero E6 (ATCC \# CRL 1586) and HEK293T (ATCC \# CRL-3216). 
Mycoplasma contamination

Commonly misidentified lines (See ICLAC register)

\section{We confirm that all cell lines were negative for mycoplasma contamination}

No commonly misidentified cell lines were used.

\section{Human research participants}

Policy information about studies involving human research participants

Population characteristics

Recruitment

Ethics oversight
COVID-19 patients involved in this study were confirmed by PCR and hospitalized. Healthy control involved in this study were recruited prior to COVID-19 outbreaks for other studies. As the study aim is for assay development, all patient information (age, gender, disease severity etc) was deidentified.

All recruitment was conducted by staff members at the National Center for Infection Diseases, Singapore, with relevant IRB approvals (detaield in the manuscript). No selection process was involved as all PCR-confirmed COVID-19 patients were recruited for this study as part of a nation-wide COVID-19 response in Singapore.

Ethics oversight for laboratory work covered by ethics committees of the Duke-NUS Medical School and National University of Singapore, and the Second Hospital of Nanjing, China.

Note that full information on the approval of the study protocol must also be provided in the manuscript. 